\title{
ON SPIRALLIKE INTEGRAL OPERATORS
}

\section{SUBHAS S. BHUSNOORMATH AND MANJUNATH V. DEVADAS}

Abstract. In this paper the integral operators

$$
F(z)=\left[(\beta+\gamma) / z^{\gamma} \int_{0}^{z}[f(t)]^{\beta} t^{\gamma-1} d t\right]^{1 / \beta}
$$

for $f(z) \in S^{\alpha}(\lambda, a, b)$ are studied. $S^{\alpha}(\lambda, a, b)$ as a subclass of the class of all spirallike functions was introduced and studied by the authors. It is shown that $F(z)$ is also in $S^{\alpha}(\lambda, a, b)$, whenever $f(z)$ is in $S^{\alpha}(\lambda, a, b)$, under certain restrictions.

\section{Introduction:}

In [1], we introduced the class $S^{\alpha}(\lambda, a, b)$ of functions $f(z)=z+\sum_{n=2}^{\infty} a_{n} z^{n}$ satisfying the condition,

$$
\frac{e^{i \alpha} z f^{\prime}(z) / f(z)-i \sin \alpha-\lambda \cos \alpha}{(1-\lambda) \cos \alpha}-a<b
$$

where $|\alpha|<\pi / 2, \lambda \in[0,1), a \in C, b \in R$ with Re $a \geq b-\lambda /(1-\lambda)$ and $|a-1|<$ b. $S^{\alpha}(\lambda, a, b)$ is the subclass of the class of all $\alpha$-spiral functions. By specializing the parameters in $S^{\alpha}(\lambda, a, b)$, we obtain various subclasses studied earlier. $S^{\alpha}(\lambda, \infty, \infty)$ is the class of spirallike functions of order $\lambda$ and $S^{\alpha}(0, \infty, \infty)$ is the class of all $\alpha$-spiral functions. Consider the following integral,

$$
F(z)=\left[(\beta+\gamma) / z^{\gamma} \int_{0}^{z}[f(t)]^{\beta} t^{\gamma-1} d t\right]^{1 / \beta}=I(f)
$$

where $f(z) \in S^{\alpha}(\lambda, a, b), \beta$ and $\gamma$ are complex numbers with $\beta=|\beta| e^{i \alpha},|\alpha|<\pi / 2$. In this paper, we show that $F(z) \in S^{\alpha}(\lambda, a, b)$, under certain restrictions.

Received September 21, 1992.

1991 Mathematics Subject Classification. Primary 30 C 45.

Key words and phrases. Spirallike functions, integral operators, subordination, univalent functions. 


\section{Perliminaries:}

It is convenient to phrase our results in terms of subordination. If $f$ and $g$ are analytic in the unit disc $E=\{z /|z|<1\}$, we say that $f$ is subordinate to $g$, written $f(z) \prec g(z)$ or $f \prec g$, if $g$ is univalent, $f(0)=g(0)$ and $f(E) \subset g(E)$. Using this concept, the functions in $S^{\alpha}(\lambda, a, b)$ can be redefined. From (8) of [1], we can write

$$
z f^{\prime}(z) / f(z) \prec \frac{b e^{i \alpha}+\left[c(1-\lambda) \cos \alpha+(1-\bar{a}) e^{i \alpha}\right] z}{\left.b e^{i \alpha}+(1-\bar{a}) e^{i \alpha}\right] z}
$$

or equivalently,

$$
e^{i \alpha} z f^{\prime}(z) / f(z) \prec \frac{e^{i \alpha}+A z}{1+B} \frac{\dot{z}}{z}
$$

where $f(z) \in S^{\alpha}(\lambda, a, b), A=\left\{c(1-\lambda) \cos \alpha+(1-\bar{a}) e^{i \alpha}\right\} / b, B=(1-\bar{a}) / b, c=b^{2}-\mid 1-$ $\left.\bar{a}\right|^{2}$ and $\alpha, \lambda, a, b$ are as above.

We need the following lemmas to prove our main theorem.

Lemma 1 [3,Corollary 1.1$]$ : Let $\beta$ and $\gamma$ be complex numbers with $\beta \neq 0$ and let $f(z)=z+a_{2} z^{2}+\ldots$, be regular in $E$. If

$$
\operatorname{Re}\left\{\beta z f^{\prime}(z) / f(z)+\gamma\right\}>0
$$

for $z \in E$, then the function $F$ defined by

$$
F^{\prime}(z)=\left[(\beta+\gamma) / z^{\gamma} \int_{0}^{z}[f(t)]^{\beta} t^{\gamma-1} d t\right]^{1 / \beta}=I(f)(z)
$$

is regular in $E, F(z) / z \neq 0$ and $\operatorname{Re}\left\{\beta z F^{\prime}(z) / F(z)+\gamma\right\}>0$.

Lemma 2 [3, Corollary 3.1]: Let $\beta$ and $\gamma$ be complex numbers with $\beta \neq 0$, and let $f(z)=z+a_{2} z^{2}+\ldots$, and $g(z)=z+b_{2} z^{2}+\ldots$, be regular functions in $E$ that satisfy

$$
z f^{\prime}(z) / f(z) \prec z g^{\prime}(z) / g(z)
$$

Let $P(z)=\beta\left\{z g^{\prime}(z) / g(z)\right\}+\gamma$ and suppose $P$ satisfies

(i) $\operatorname{Re} P(z)>0$

and

(ii) $P$ and $1 / P$ are convex (univalent) in $E$.

If $E \equiv I(f)$ and $G \equiv I(g)$, where $I$ is given by (2.3) then $F$ and $G$ are regular in $E, z G^{\prime}(z) / G(z)$ is univalent in $E$ and

$$
z F^{\prime}(z) / F(z) \prec z G^{\prime}(z) / G(z) \prec z g^{\prime}(z) / g(z)
$$

Note that the condition (i) of Lemma 2 and Lemma 1 imply that $G$ is regular in $E$ with $G(z) / z \neq 0$. Also from (i) and (2.4), we have $\operatorname{Re}\left\{\beta\left[z f^{\prime}(z) / f(z)\right]+\gamma\right\}>0$. Which together with Lemma 1 implies that $F$ is regular in $E$ and $F(z) / z \neq 0$. 


\section{Now we prove our main theorem.}

Theorem $\mathbb{1}$ : Let $\beta$ and $\gamma$ be complex numbers with $\operatorname{Re} \beta>0$, satisfying conditions,

$$
\operatorname{Re}\left\{\bar{B} \beta A e^{-i \alpha}+|B|^{2} \gamma+\beta A e^{-i \alpha}-\beta B\right\}>0
$$

and

$$
\left|\beta A e^{-i \alpha}+B \gamma\right|<|\beta+\gamma|
$$

where $A=\left\{c(1-\lambda) \cos \alpha+(1-\bar{a}) e^{i \alpha} / b, B=(1-\bar{a}) / b, c=b^{2}-|1-\bar{a}|^{2}\right.$. If $\beta=|\beta| e^{i \alpha}$ and the functions $f(z)=z+a_{2} z^{2}+\ldots, g(z)=z+b_{2} z^{2}+\ldots$ are regular in $E$ satisfying

$$
e^{i \alpha} \frac{z f^{\prime}(z)}{f(z)} \prec \frac{e^{i \alpha}+A z}{1+B z} \equiv e^{i \alpha} \frac{z g^{\prime}(z)}{g(z)}
$$

Then the functions

$$
F(z)=I(f)(z)=\left[\frac{\beta+\gamma}{z^{\gamma}} \int_{0}^{z}\{f(t)\}^{\beta} \cdot t^{\gamma-1} \cdot d t\right]^{1 / \beta}
$$

and $G(z)=I(g)(z)$ are regular in $E$ and satisfy

$$
e^{i \alpha} \frac{z F^{\prime}(z)}{F(z)} \prec e^{i \alpha} \frac{z G^{\prime}(z)}{G(z)} \prec e^{i \alpha} \frac{z g^{\prime}(z)}{g(z)}
$$

Proof. The function $g$ defined by (3.3) is given by

$$
g(z)=z(1+B z)^{\left(A / B e^{-i \alpha}-1\right.}=z+b_{2} z^{2}+\ldots
$$

(all powers are principal powers) and satisfies

$$
z f^{\prime}(z) / f(z) \prec z g^{\prime}(z) / g(z)
$$

Now let

$$
\begin{aligned}
P(z) & =\beta\left\{\frac{z g^{\prime}(z)}{g(z)}\right\}+\gamma \\
& =\frac{(\beta+\gamma)+\left(\beta A e^{-i \alpha}+B \gamma\right) z}{1+B z}
\end{aligned}
$$

Putting $\left(\beta A e^{-i \alpha}+B \gamma\right) /(\beta+\gamma)=\rho$, we get,

$$
P(z)=\{(\beta+\gamma)(1+\rho z)\} /(1+B z)
$$


(3.7) is a linear transformation which maps $|z|<1$ on to a circle with centre at

$$
\frac{(\beta+\gamma)-\bar{B}\left(\beta A e^{-i \alpha}+B \gamma\right)}{\left(1-|B|^{2}\right)} \quad \text { and }
$$

radius as

$$
\left|\beta A e^{-i \alpha}+B \gamma-(\beta+\gamma) \bar{B}\right| /\left(1-|B|^{2}\right),
$$

which implies that $\operatorname{Re} P(z) \geq 0$ if and only if (3.1) is satisfied. $P(z)$ and $1 / P(z)$ are convex if $|B|<1$ and (3.2) is satisfied. Thus $P$ satisfies all the conditions of Lemma 2, and (3.5) is proved.

Remark. (1) For $a=b=\infty$, the condition (3.1) reduces to the condition (5) of Theorem 1 of [2].

(2) (3.5) essentially proves $G$ is the best dominant over the class of integral operators of functions in $S^{\alpha}(\lambda, a, b)$ and is given by

$$
\frac{z G^{\prime}(z)}{G(z)}=\frac{1}{\beta}\left[\frac{z^{\beta+\gamma}(1+B z)^{\left(\frac{\beta A e^{-i \alpha}}{\beta}-\beta\right.}-\beta}{\int_{0}^{z} t^{\beta+\gamma-1}(1+B t)^{\left(\left\{\beta A e^{-i \alpha} / B\right\}-\beta\right)} d t}-\gamma\right]
$$

\section{References}

[1] S.S. Bhoosnurmath and M.V. Devadas, "Subclasses of $\alpha$-spiral functions," Ganita, 46, No.2, 1994 (to appear).

[2] S.S. Miller and P.T. Mocanu, "On a class of spirallike integral operators", Rev Roumaine Math. Pures Appl., 31 (1986), 225-230.

[3] S.S. Miller and P.T. Mocanu, "Univalent solutions of Briot-Bouquet differential subordinations," J. Differential equations, 56 (3) (1985), 297-309.

Department of Mathematics, Karnatak University, Dharwad - 580 003, INDIA.

Department of Mathematics, B.V.B. College of Engg. \& Tech, Hubli - 580 031, INDIA. 